\title{
Ultraschall - Etablierte Methodik oder innovative Messtechnik?
}

\author{
Michael Metzenmacher ${ }^{1}$, Alexander Beugholt ${ }^{1}$, Dominik Geier ${ }^{1}$, Prof. Dr. Thomas Becker ${ }^{1}$ \\ ${ }^{1}$ Lehrstuhl für Brau- und Getränketechnologie, TU München, Freising \\ Kontakt: dominik.geier@tum.de
}

\section{Einleitung}

Die Online-Analytik ist eine wesentliche Grundlage für die Automatisierung und Digitalisierung von Produktionsprozessen [1-3]. Gerade der Einsatz von Ultraschalltechniken hat sich in verschiedenen Bereichen der Prozessüberwachung in den letzten Jahren deutlich erhöht. Im Vergleich zu anderen vorhandenen Techniken bietet Ultraschall eine schnelle, kostengünstige und nicht-destruktive Methode zur Analyse von Prozess- und Produktparametern [4-6]. Durch ihren nicht-invasiven Charakter bieten sich vielfältige Anwendungsgebiete, gerade in den produktsensiblen Prozessschritten der Life Sciences [7-9] und der Lebensmittelindustrie [10-12].

In diesem Beitrag werden zunächst die Grundlagen und der Status Quo im Bereich der messtechnischen Anwendung von Ultraschall dargestellt. Anschließend werden Applikationsbeispiele, welche derzeit am Lehrstuhl für Brauund Getränketechnologie beforscht werden, vorgestellt und erörtert. Aktuelle Untersuchungen des Lehrstuhles für Brau- und Getränketechnologie der Technischen Universität München beschäftigen sich u.a. mit der nicht-invasiven Bestimmung kleinster Füllstände in Flüssigkeitstanks. Dazu wird eine Kombination aus Time-of-Flight-Messungen (TOF) und Oberflächenwellen-Analyse genutzt. Dieser Ansatz genügt höchsten Ansprüchen an das Hygienic Design und ermöglicht auch eine Messung an gekrümmten Oberflächen. Ein weiterer Anwendungsfall für Ultraschall ist die Überwachung der Ausbildung von Fouling, das Monitoring der Entfernung der Foulingschicht beim Cleaning in Place sowie die anschließende Reinigungskontrolle. Auch zur Kontrolle der alkoholischen Gärung kann Ultraschall eingesetzt werden. Aus den Ultraschallsignalen werden hier mittels chemometrischer Analyse so genannte UltraschallFeatures extrahiert, welche eine Korrelation mit Edukten und Produkten der Fermentation erlauben.

\section{Messmethoden}

Die ultraschallbasierte Messung verschiedener Prozessgrößen kann durch unterschiedliche Methoden realisiert werden, siehe Tabelle 1. Die Generierung der Ultraschallwellen wird dabei meist durch piezokeramische Transducer realisiert, welche elektrische Energie in mechanische Energie umwandeln und umgekehrt. Ultraschall-Transducer können in einem weiten Temperaturbereich eingesetzt werden (bis zu $300{ }^{\circ} \mathrm{C}$ ) und besitzen die erforderliche Langzeitstabilität und Zuverlässigkeit für industrielle Anwendungen [13]. Dabei können entsprechend den Anforderungen spezifische Keramiken zur Konstruktion der Transducer verwendet werden [14]. Als Ultraschall bezeichnet man mechanische Wellen mit Frequenzen über $16 \mathrm{kHz}$ und somit oberhalb des Hörbereichs des Menschen [15]. Je nach Medium können sich Schallwellen unterschiedlich ausbreiten und werden in Longitudinal-, Transversal- und Oberflächenwellen unterteilt [16]:

Longitudinalwellen (LW): Bei LW ist die Schwingungsrichtung der einzelnen Teilchen mit der Ausbreitungsrichtung der Schallwelle identisch. Während der Wellenausbreitung bilden sich Kompressions- und Dekompressionsbereiche aus. LW breiten sich in Gasen und Flüssigkeiten aus.

Transversalwellen (TW): Bei TW ist die Partikelbewegung senkrecht zur Wellenausbreitung. Die Schallgeschwindigkeit ist im Gegensatz zu den LW deutlich verringert. TW können sich aufgrund ihrer Charakteristik zudem nur in Medien mit Schermodul, also Festkörpern, ausbilden.

Oberflächenwellen (OW): OW bestehen aus einer Kombination von Longitudinal- und Transversalwellen und breiten sich insbesondere an den Grenzflächen zwischen den Phasen fest/flüssig und fest/gasförmig aus. Die Schallgeschwindigkeit der OW ist dabei vergleichbar mit den TW.

Tabelle 1: Ultraschallbasierte Messmethoden, genutzte Schallparameter und typische Einsatzgebiete.

MESSMETHODE PARAMETER EINSATZGEBIETE

\begin{tabular}{|c|c|c|}
\hline REFLEXION & $\begin{array}{l}\text { TOF } \\
\text { Frequenz } \\
\text { Amplitude } \\
\text { Impedanz }\end{array}$ & $\begin{array}{l}\text { Abstand, Füllhöhe, Schallgeschwindigkeit, Position, } \\
\text { Objektstruktur, NDT (zerstörungsfreie Prüfung), } \\
\text { Dichte, Viskosität, Konzentration, } \\
\text { Bewegung, Fließgeschwindigkeit }\end{array}$ \\
\hline TRANSMISSION & $\begin{array}{l}\text { TOF } \\
\text { Frequenz } \\
\text { Amplitude }\end{array}$ & $\begin{array}{l}\text { Konzentrationen, } \\
\text { Partikelgrößenverteilung in (gering dämpfenden) Emulsionen } \\
\text { und Suspensionen, Schallgeschwindigkeit, } \\
\text { Fließgeschwindigkeit, Dichte, Viskosität, Temperatur }\end{array}$ \\
\hline EMISSION & $\begin{array}{l}\text { Frequenz } \\
\text { Amplitude }\end{array}$ & Prozessüberwachung \\
\hline
\end{tabular}




\section{Reflexionsmessung}

Reflexionsmessungen können sowohl mit einem (PulsEcho-Modus) als auch mit zwei Transducern (Pitch-CatchModus) realisiert werden, siehe Abbildung $1 \mathrm{a}$ ) und b). Dabei wird die Ultraschallwelle durch den Sender in das Medium eingekoppelt und die Reflexion der Wellen an Grenzflächen durch den Empfänger aufgezeichnet. Die Ultraschallwelle durchläuft das Medium daher immer zweimal. Die Messmethode ermöglicht sowohl die Bestimmung der Schalllaufzeit (TOF) als auch der Dämpfung (Amplitude), Frequenz und Impedanz (Z). Die akustische Impedanz $Z$ berechnet sich dabei aus der Dichte und der Schallgeschwindigkeit des Mediums:

$$
Z=\frac{p}{u}=\rho c
$$

$p$ : Schalldruck, $u$ : Schallschnelle, $\rho$ : Dichte, $c$ : Ultraschallgeschwindigkeit, Z: Impedanz.

An Grenzflächen zwischen zwei Medien mit unterschiedlicher Impedanz kommt es dabei zu Reflexionen. Der Reflexionskoeffizient $R$ ist dabei das Verhältnis der emittierten Schallenergie $p_{e}$ zur detektierten Schallenergie $p_{d}$ und ermöglicht Rückschlüsse auf die Eigenschaften der durchschallten Medien:

$$
R=\frac{p_{d}}{p_{e}}=\frac{Z_{2}-Z_{1}}{Z_{2}+Z_{1}}
$$

$Z_{1}$ : akustische Impedanz Medium 1, $Z_{2}$ : akustische Impedanz Medium 2

\section{Transmissionsmessung}

Bei Transmissionsmessungen, siehe Abbildung $1 \mathrm{c}$ ), wird das Ultraschallsignal durch einen Transducer emittiert (Sender) und durch einen weiteren Transducer aufgezeichnet (Empfänger). Die Schallwelle durchdringt dabei das Medium genau einmal. Diese Messmethode ermöglicht die Bestimmung der Schalllaufzeit (TOF), Dämpfung (Amplitude) und Frequenz. Die Eigenschaften der durchschallten Medien beeinflussen dabei die Ultraschallwelle und damit das erhaltene Signal. Auch Oberflächenwellen können so aufgezeichnet und analysiert werden, siehe Abbildung $1 \mathrm{e}$ ).

\section{Emissionsmessung}

Bei der Emissionsmessung werden Schallsignale aufgezeichnet, welche durch den Prozess selbst emittiert werden, siehe Abbildung $1 \mathrm{~d}$ ). Änderungen im Prozess beeinflussen die Schallsignale im Zeit- und Frequenzbereich. Diese Signaländerungen werden detektiert und charakterisieren den Prozess.

Die unterschiedlichen Messmethoden nutzen dabei jedoch meist dieselben Schallparameter, siehe Tabelle 1. Die Tabelle verdeutlicht die Herausforderungen bei ultraschallbasierten Messungen. Während wenige Schallparameter bestimmt werden, gibt es eine Vielzahl an Applikationen, welche durch die Änderung der Parameter charakterisiert werden können. Durch intelligente Auswertung der Sensorsignale und Modellbildung können Korrelationen mit physikalischen und (bio-)chemischen Parametern aufgestellt werden. Weiterhin können die Modelle durch die Integration zusätzlicher Parameter (Druck, Temperatur, etc.) erheblich verbessert werden.

\section{Anwendungsgebiete}

Durch die Flexibilität der ultraschallbasierten Messung ergeben sich viele Anwendungsgebiete, welche die Vorteile einer nicht-invasiven und kostengünstigen Messung nutzen. Insbesondere im Bereich der Lebensmittelproduktion und der Life Sciences gewinnen inline- und echtzeitfähige Sensorkonzepte ohne Produktberührung an Bedeutung.

\section{Temperaturmessung}

Da sich die physikalischen Eigenschaften von Materialien (akustischer Widerstand, Dichte, etc.) in Abhängigkeit der Temperatur stark verändern, werden Ultraschallsignale durch Temperaturschwankungen beeinflusst. Durch den Einsatz von definierten Vorlaufstrecken (mit bekannten Schalleigenschaften), kann mittels Auswertung des ersten Echos und der Messung der Schalllaufzeit (TOF) die

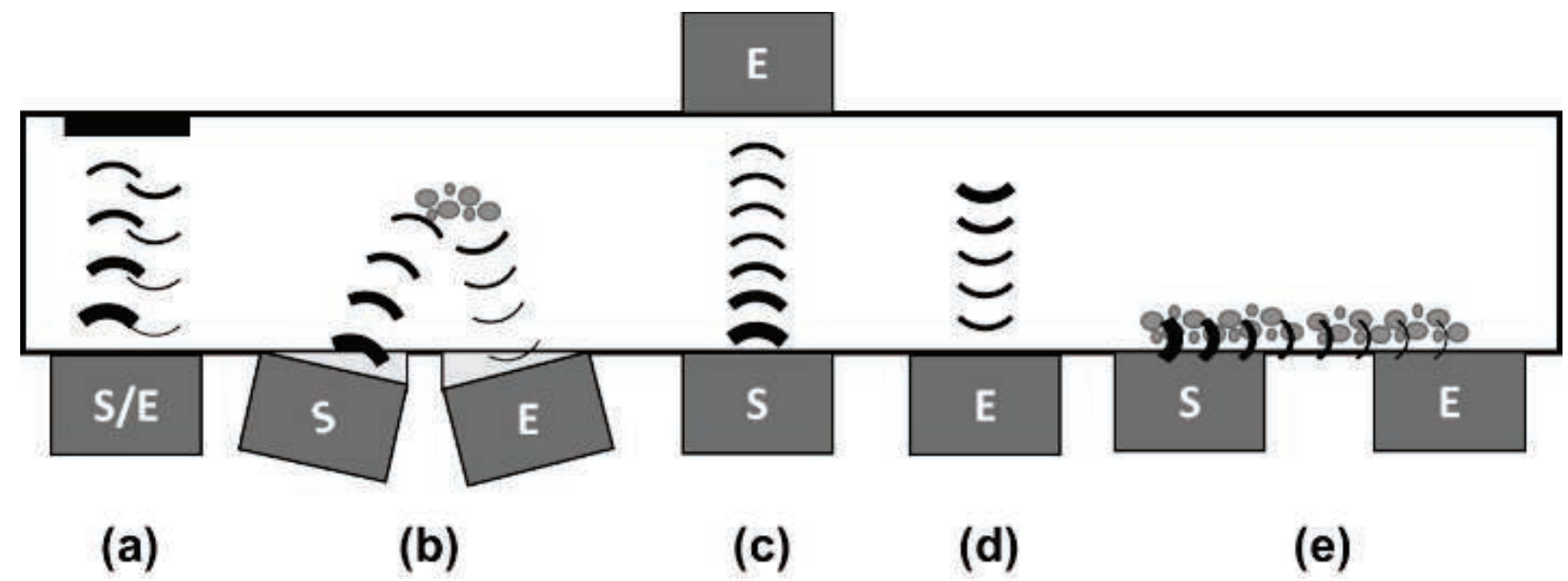

Abbildung 1: Ultraschallbasierte Messanordnungen mit Sender (S) und Empfänger (E). a) Puls-Echo-Modus, b) Pitch-CatchModus, c) Transmission, d) Emission, e) Oberflächenwellenmessung im Pitch-Catch-Modus. 
Schallgeschwindigkeit bestimmt werden. Diese ist von den bekannten Materialeigenschaften der Vorlaufstrecke abhängig und ermöglicht somit Rückschlüsse auf die vorherrschende Temperatur.

\section{Füllstandsbestimmung}

Die Bestimmung von kleinen und großen Füllständen ist essenziell für viele industrielle Prozesse. Dazu werden aktuell sowohl mechanische Messprinzipien (Schwimmer, Differenzdruck, etc.), Leitfähigkeitsmessungen, kapazitive Messungen, optische Methoden, Mikrowellen, Radar und Ultraschall genutzt. Aufgrund ihrer Invasivität sind jedoch besonders im Lebensmittel- und Life Science-Bereich viele Messmethoden nicht geeignet. Die Füllstände von festen und flüssigen Medien können durch den Einsatz von Luftultraschall bestimmt werden, siehe Abbildung 2 a). Dabei wird die Ultraschallwelle über die Luft übertragen und an der Grenzfläche reflektiert. Der Einsatz geeigneter Schallfrequenzen ermöglicht präzise Messungen sowohl für große als auch für kleine Füllstände [17]. Die Füllstandsbestimmung von Medien, die eine Schaumbildung zeigen - bspw. bei Fermentationsprozessen - ist mit dieser Methode jedoch nicht realisierbar, da die Ultraschallwellen in diesem Fall vom Schaum reflektiert werden. Ultraschallbasierte Messungen von schäumenden Medien sollten daher durch eine Messung „Von unten“ realisiert werden. Bei dieser Messung wird der Ultraschall nicht durch die Luft, sondern durch die Tankwand in die Flüssigkeit übertragen und an der Phasengrenze (Flüssigkeit/Luft) reflektiert, siehe Abbildung 2 b). Dadurch entstehen in der Tankwand zusätzliche Reflexionen, welche die Messung von kleinen Füllständen im Puls-Echo-Modus stark behindern. Größere Füllstände in Tanks können so jedoch mit einem Transducer realisiert werden $[18,19]$. Derzeit in Industrie und Forschung verbreitete Systeme nutzen für die Füllstandsbestimmung überwiegend Longitudinalwellen. Ein neuer Ansatz zur Kombination von Longitudinal-, Transversal- und Oberflächenwel- len zur Füllhöhenbestimmung wird von Raja et. al. [20] vorgestellt. Über einen Hohlleiter wird das Ultraschallsignal in das Medium eingekoppelt und im Puls-Echo-Modus aufgezeichnet. Durch die Laufzeitunterschiede (unterschiedliche Schallgeschwindigkeiten) der einzelnen Wellentypen, kann jeder Wellentyp einzeln sowohl in der Zeit- als auch der Frequenzdomäne analysiert werden. Diese Methode ist jedoch invasiv und kann daher in vielen Prozessen nicht eingesetzt werden. Die nicht-invasive Messung kleinster Füllstände in geschlossenen Tanks kann auch durch die Kombination aus Time-of-Flight-Messungen und OberflächenwellenAnalyse realisiert werden. Die Ultraschallwellen werden in einem definierten Winkel eingekoppelt und breiten sich sowohl im Medium als auch in der Tankwand aus, siehe Abbildung $2 \mathrm{c}$ ). Die Oberflächenwellen, welche sich an der Grenzfläche zwischen Medium und Tankinnenwand ausbilden, werden dabei durch das Medium gedämpft. Bei der Änderung der Füllhöhe zwischen den beiden Transducern ändert sich daher auch die Dämpfung. Durch den kombinierten Einsatz von Oberflächenwellen für kleinste Füllstände und der Time-of-Flight-Messung für größere Füllstände, können Tanksysteme nicht-invasiv und berührungslos mittels Ultraschall überwacht werden.

\section{Fouling-Detektion}

Sprühtrocknungsprozesse spielen als schonende Trocknungstechniken in vielen Industrien für die Herstellung von hochwertigen, pulverförmigen Produkten eine herausragende Rolle. Durch die thermische Belastung bilden sich jedoch Produktablagerungen - Fouling genannt - an den Gerätewänden aus. Bekannte Möglichkeiten Fouling in diesen meist CIP-fähigen Anlagen zu detektieren basieren auf Messungen von elektrischer Impedanz, Wärmedurchgangskoeffizienten, -kapazitäten sowie die Messung elektrischer oder thermischer Widerstände. Aufgrund von mangelnder Empfindlichkeit oder der Invasivität werden diese Methoden aktuell kaum in der industriellen Praxis ein-

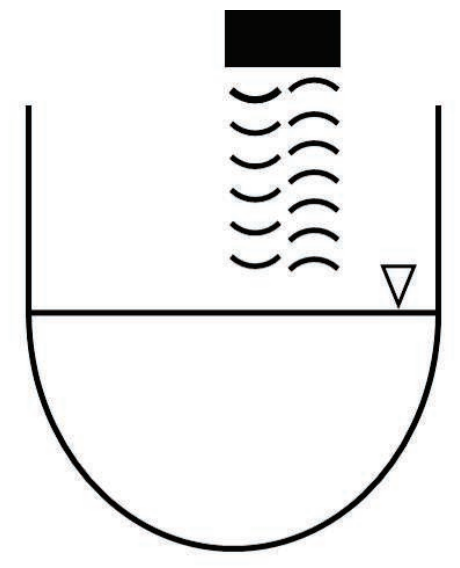

(a)

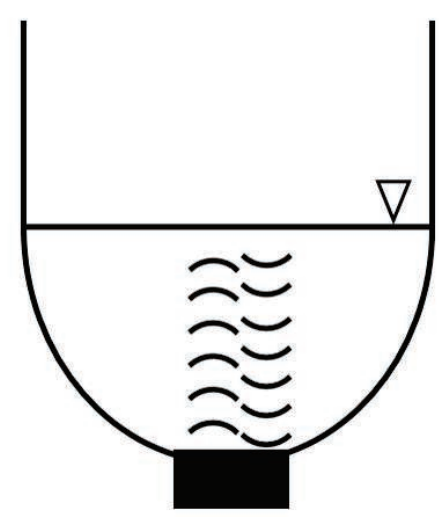

(b)

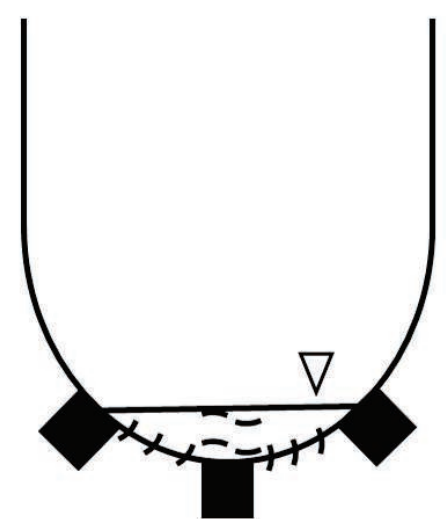

(c)

Abbildung 2: Ultraschallbasierte Füllstandsbestimmung. a) Füllstandsbestimmung mit Luftultraschall, b) Füllstandsbestimmung mit Longitudinalwellen (Puls-Echo-Modus), c) Kombinierte Füllstandsbestimmung mit Longitudinal- und Oberflächenwellen. 
gesetzt. Das Prinzip der Fouling-Überwachung mittels Ultraschall basiert auf der Änderung der akustischen Impedanz, welche von den Eigenschaften der ausgebildeten Fouling-Schicht abhängt. Durch die Messung im PulsEcho-Modus können über die Bestimmung des Reflexionskoeffizienten materialspezifische Informationen der Grenzschicht ermittelt werden. Das grundlegende Messsystem ist in Abbildung 3 dargestellt.

Die Ultraschallsignale werden sowohl in der Zeit- als auch in der Frequenzdomäne, nach diskreter Fourier-Transfor-

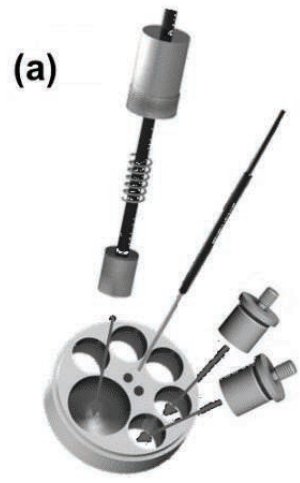

(b)

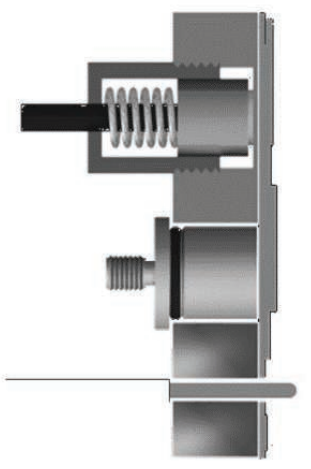

Abbildung 3: a) Schematische Darstellung des ultraschallbasierten Messaufbaus, b) Adaption des Messaufbaus an den Sprühturm [18].

mation (DTF), analysiert und ausgewertet. Störgeräusche aufgrund von Pumpenvibrationen etc. werden mittels Frequenz-Bandpassfiltern eliminiert. Weitere zeitbasierte $\mathrm{Pa}-$ rameter der Ultraschallsignale (Signalenergie, Signaldämpfung, etc.), wie auch frequenzabhängige Parameter (Frequenzverschiebung etc.) erweitern die Auswertung der Ultraschallsignale. Auch die Temperatur hat einen starken Einfluss auf die Ultraschallausbreitung, weshalb die Prozesstemperatur überwacht und aufgezeichnet wird. Referenzmessungen mittels REM (Rasterelektronenmikroskop) erlauben dabei eine externe Referenzüberwachung der Foulingbildung. Mittels Partial Least Squares Regression (PLSR) wurde der Zusammenhang zwischen den Referenzmessungen und den Ultraschallmessungen ermittelt, siehe Abbildung 4.

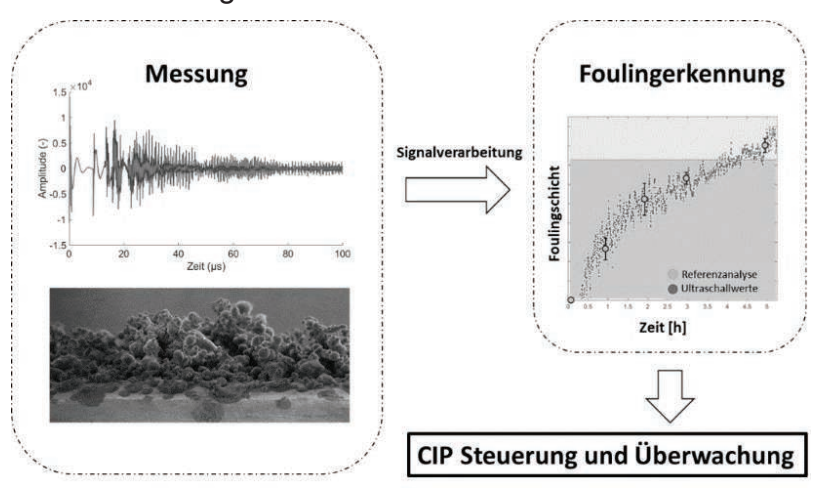

\section{Abbildung 4: Überwachung der Foulingbildung bei der Sprühtrocknung von Milch.}

Die entwickelte ultraschallbasierte Methode erlaubt dabei sowohl die Fouling-Überwachung während der Sprühtrocknung von Milch [21,22] als auch die Detektion von Milch-
Fouling im Verarbeitungsprozess $[23,24]$ und kann zur Charakterisierung von Fouling-Schichten [25] sowie zur Überwachung der Reinigung $[26,27]$ genutzt werden. Hierbei wurden sowohl qualitative als auch quantitative Aussagen über Reinigungsintensität und -erfolg getroffen. So konnten Reinigungsintervalle optimiert und Chargen- bzw. Produktverschleppung verhindert werden.

\section{Fermentationsüberwachung}

Des Weiteren kann die alkoholische Gärung durch ultraschallbasierte Messungen überwacht werden [28]. Der Abbau von Extrakt und die damit einhergehende Produktion von Ethanol und Kohlenstoffdioxid verändern die akustischen Eigenschaften des Mediums. Die Abnahme der Dichte des Mediums mit fortschreitender Fermentation bedingt eine Änderung der Impedanz an der Grenzfläche sowie eine Änderung der Ultraschallgeschwindigkeit. Auch die Dämpfung der Ultraschallsignale wird sowohl durch die Dichteunterschiede als auch durch die Bildung von Kohlenstoffdioxid verstärkt. Ultraschall ermöglicht zudem die Bestimmung von mehr als einer Eigenschaft eines Stoffsystems ohne Verdünnungsschritte und Probennahme. Eine schematische Darstellung der ultraschallbasierten Bestimmung von Prozessparametern während der Fermentation ist in Abbildung 5 dargestellt. Die Auswertung erfasst physikalische Parameter und spezifische, signalbasierte Änderungen im Ultraschallsignal. Durch dieses Vorgehen konnte durch Bestimmung des Absorptionskoeffizienten eine Korrelation zur Hefezellzahl [29] hergestellt sowie kritische Prozessparameter während der alkoholischen Gärung [30,31] bestimmt werden. Beispielsweise konnte der Extraktabbau und die Produktion von Ethanol online verfolgt werden.

\section{Herausforderungen und Potential}

Der Einsatz von Ultraschall bietet eine Vielzahl an Anwendungen in der Prozessindustrie. Während es bereits Einsatzgebiete mit hoher Akzeptanz für Ultraschall gibt (z. B. Füllstandsbestimmung), werden Ultraschallsensoren trotz ihres großen Potentials nur im geringeren Maße für die Prozesskontrolle eingesetzt. Ultraschallbasierte Messsysteme finden ihre bevorzugte Anwendung, wenn der Einsatz von etablierten Systeme aufgrund von prozesstechnischen Begebenheiten nicht möglich ist. Grundvoraussetzung für den erfolgreichen Einsatz von Ultraschall als Messtechnik ist das Vorhandensein von akustisch transparenten Medien sowie von Informationen über die akustischen Eigenschaften der untersuchten Medien bzw. Stoffsysteme. Zudem ist aufgrund vieler bestimmbarer Schallparameter und der zahlreichen Einflussfaktoren auf die Ultraschallsignale eine komplexe Signalverarbeitung essentiell. 


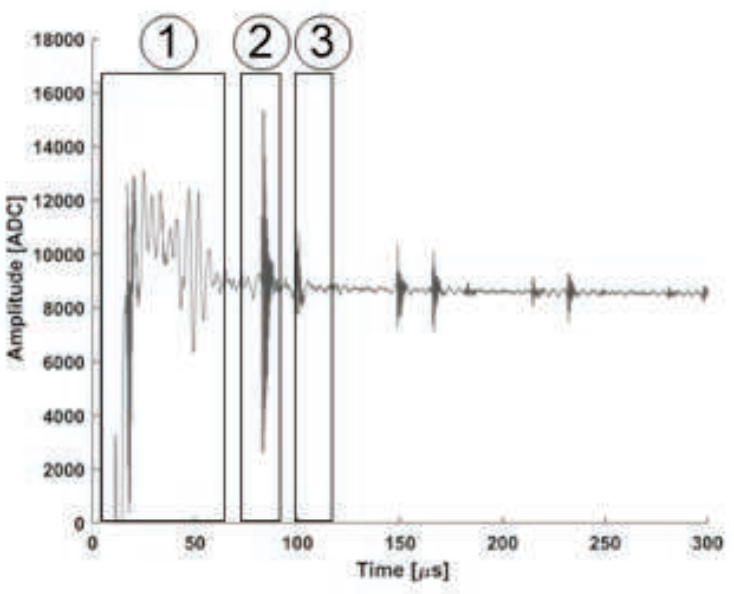

(a)

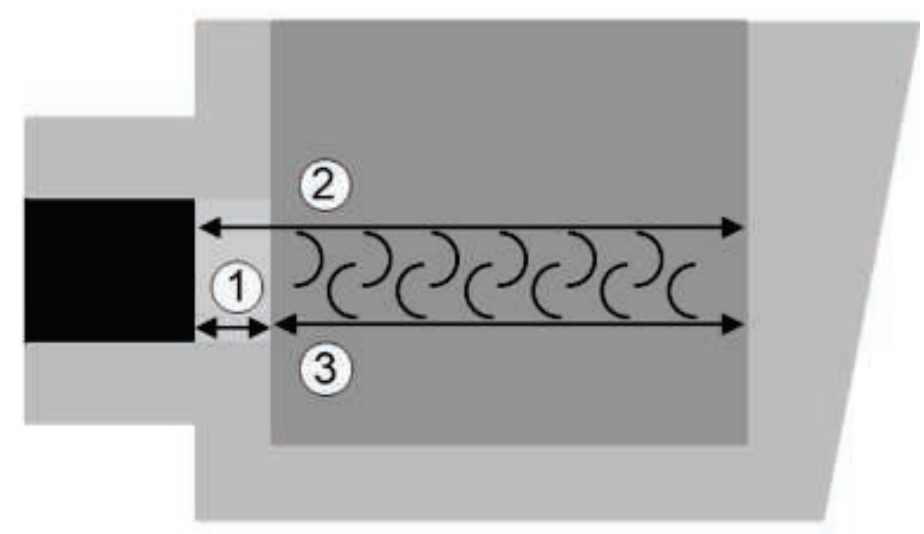

(b)

Abbildung 5: Schematische Darstellung der ultraschallbasierten Bestimmung von Prozessparametern während der Fermentation. a) Signal eines Ultraschallsensors für die Fermentationskontrolle mit (1) Impedanz-Signal, (2) 1. Echo,

(3) 2. Echo, b) Ultraschallsensoreinheit mit schematischer Darstellung der Ultraschallreflexionen (1), (2) und (3).

Ultraschall bietet aber bei geringem Energieverbrauch eine Messtechnik, die online zur Bestimmung zahlreicher Prozessparameter eingesetzt werden kann. Da UltraschallSensoren keinen direkten Kontakt zum Medium benötigen sind sie nicht-invasiv und die Reinigbarkeit der Produktionsanlagen wird nicht beeinflusst, was das Kontaminationsrisiko minimiert. Ultraschall-Sensoren bieten zudem eine robuste Messmethode bei gleichzeitig hoher Auflösung und können mit etablierten Messmethoden kombiniert werden, um das Potenzial vom Ultraschall voll auszuschöpfen. Insbesondere im Hinblick auf Konzepte der Industrie 4.0 und KI-gestützter Systeme ermöglichen ultraschallbasierte Sensoren innovative Ansätze für die industrielle Implementierung.

\section{Danksagung}

Das IGF-Vorhaben AiF $18754 \mathrm{~N}$ des Forschungskreises der Ernährungsindustrie e.V. (FEI) wurde über die AiF im Rahmen des Programms zur Förderung der Industriellen Gemeinschaftsforschung (IGF) vom Bundesministerium für Wirtschaft und Energie aufgrund des Beschlusses des Deutschen Bundestages gefördert. Das Projekt Wissensbasierte Prozessintelligenz Projektnummer 031A616D wurde durch das Bundesministerium für Bildung und Forschung gefördert. Die Forschungsvorhaben FKZ 4025009PR6 und FKZ 4021103 des zentralen Innovationsprogramms Mittelstand (ZIM) wurden mit der Unterstützung der AiF Projekt GmbH durchgeführt und vom Bundesministerium für Wirtschaft und Energie gefördert.

\section{Literatur}

[1] L. Yang, "Industry 4.0: A survey on technologies, applications and open research issues," Journal of Industrial Information Integration, vol. 6, pp. 1-10, 2017.
[2] L. D. Xu, E. L. Xu, and L. Li, "Industry 4.0: state of the art and future trends," International Journal of Production Research, vol. 56, no. 8, pp. 2941-2962, 2018.

[3] K. Zhou, T. Liu, and L. Zhou, "Industry 4.0: Towards future industrial opportunities and challenges," in 2015 12th International Conference on Fuzzy Systems and Knowledge Discovery: FSKD 2015 : 15-17 August, Zhangjiajie, China, Z. Tang, Ed., pp. 21472152, IEEE, Piscataway, NJ, 2015.

[4] Beugholt, A., Metzenmacher, M., Geier, D., Becker, T., "Ultraschall - eine nichtinvasive Online-Messtechnik," Der Weihenstephaner, no. 3, pp. 118-120, 2019.

[5] T. Stelzer, D. Pertig, and J. Ulrich, "Ultrasonic crystallization monitoring technique for simultaneous in-line measurement of liquid and solid phase," Journal of Crystal Growth, vol. 362, pp. 71-76, 2013.

[6] R. Kotzé, R. Haldenwang, V. Fester et al., "In-line rheological characterisation of wastewater sludges using non-invasive ultrasound sensor technology," Water SA, vol. 41, no. 5, pp. 683-690, 2015.

[7] X. Chen, M. Hussein, and T. Becker, "Determination of bubble size distribution in gas-liquid two-phase systems via an ultrasound-based method," Engineering in Life Sciences, vol. 17, no. 6, pp. 653-663, 2017.

[8] S. Hoche, M. A. Hussein, and T. Becker, "Ultrasoundbased density determination via buffer rod techniques: a review," Journal of Sensors and Sensor Systems, vol. 2, no. 2, pp. 103-125, 2013.

[9] S. Hoche, M. A. Hussein, and T. Becker, "Density, ultrasound velocity, acoustic impedance, reflection and absorption coefficient determination of liquids via multiple reflection method," Ultrasonics, vol. 57, pp. 6571, 2015.

[10] Metzenmacher, M., Geier, D., Becker, T., "Ultrasound-based monitoring of cereal-based foams," The Future of Baking - Science - Technique - Technology. Hamburg: f2m (2018), pp. 22-27, 2018. 
[11] Metzenmacher M., Geier D., Becker, T., "Getreidebasierte Schäume - Lufteintrag per Ultraschall messen," brot+backwaren, no. 6, pp. 50-53, 2018.

[12] Metzenmacher, M., Geier, D., Becker, T., "Ultraschallbasierte Messung des eingebrachten Luftvolumens," Chleb + Wipetschka, 2019.

[13] P. Hauptmann, N. Hoppe, and A. Puettmer, "Ultrasonic sensors for process industry," in 2001 IEEE UItrasonics Symposium: Proceedings : an international symposium : October 7-10, 2001, Omni Hotel, Atlanta, Georgia, D. E. Yuhas and S. C. Schneider, Eds., pp. 369-378, IEEE, Piscataway, N.J, 2001.

[14] Q. X. Chen and P. A. Payne, "Industrial applications of piezoelectric polymer transducers," Measurement Science and Technology, vol. 6, no. 3, pp. 249-267, 1995.

[15] W. Beitz and K.-H. Grote, eds., Dubbel: Taschenbuch für den Maschinenbau, Springer Berlin Heidelberg, Berlin, Heidelberg, s.I., 1997.

[16] W. M. Alobaidi, E. A. Alkuam, H. M. Al-Rizzo et al., "Applications of Ultrasonic Techniques in Oil and Gas Pipeline Industries: A Review," American Journal of Operations Research, vol. 05, no. 04, pp. 274-287, 2015.

[17] S. L. Mohammed, A. Al-Naji, M. M. Farjo et al., "Highly Accurate Water Level Measurement System Using a Microcontroller and an Ultrasonic Sensor," IOP Conference Series: Materials Science and Engineering, vol. 518, no. 4, p. 42025.

[18] W. Richter, "Nicht-invasive Inhaltsmessung in Drucktanks"," Brauwelt, vol. 2016, no. 50, pp. 1483-1485, 2016.

[19] W. Richter, "Was ist noch drin? Verlässliches Volumenmanagement," Brauwelt, vol. 159, 40-41, pp. 1168-1171, 2019.

[20] N. Raja, K. Balasubramaniam, and S. Periyannan, "Ultrasonic waveguide based level measurement using flexural mode $F(1,1)$ in addition to the fundamental modes," The Review of scientific instruments, vol. 90, no. 4, p. 45108, 2019.

[21] Úbeda, M., Whitehead, I., Frankl, M., Herfellner, M., Geier, D., Becker, T., "Spray drying of milk - Ultrasonic Fouling monitoring," International Dairy Magazine, no. 8, pp. 22-24, 2018.

[22] Úbeda, M., Whitehead, I., Frankl, M., Herfellner, M., Geier, D., Becker, T., "Ultraschallbasierte FoulingÜberwachung während der Sprühtrocknung," Molkerei-Industrie, no. 7, pp. 16-19, 2018.

[23] E. Wallhäußer, W. B. Hussein, M. A. Hussein et al., "Detection of dairy fouling: Combining ultrasonic measurements and classification methods," Engineering in Life Sciences, vol. 13, no. 3, pp. 292-301, 2013.

[24] Wallhäußer, E., Hussein, W.B., Hussein, M.A., Hinrichs, J., Becker, T., "Determination of fouling presence and absence using a combined method of ultrasonic measurements and classification methods," Engineering in Life Science, vol. 13, pp. 292-301, 2013.
[25] J. Escrig, E. Woolley, S. Rangappa et al., "Clean-inplace monitoring of different food fouling materials using ultrasonic measurements," Food Control, vol. 104, pp. 358-366, 2019.

[26] E. Wallhäußer, A. Sayed, S. Nöbel et al., "Determination of cleaning end of dairy protein fouling using an online system combining ultrasonic and classification methods," Food and Bioprocess Technology, vol. 7, no. 2, pp. 506-515, 2014.

[27] Wallhäußer, E., Sayed, A.,Nöbel, S., Hussein, M.A., Hinrichs, J., Becker, T., "Determination of cleaning end of protein fouling using an online system combining ultrasonic and classification methods," Food and Bioprocess Technology, vol. 1, 01. Okt, 2013.

[28] S. Hoche, W. B. Hussein, M. A. Hussein et al., "Timeof-flight prediction for fermentation process monitoring," Engineering in Life Sciences, vol. 11, no. 4, pp. 417-428, 2011.

[29] D. Geier, K. Heermann, M. Hussein et al., "Effects of yeast and maltose concentration on ultrasonic velocity and attenuation coefficient and its application for process monitoring," Engineering in Life Sciences, vol. 14, no. 4, pp. 433-441, 2014.

[30] S. Hoche, D. Krause, M. A. Hussein et al., "Ultrasound-based, in-line monitoring of anaerobe yeast fermentation: model, sensor design and process application," International Journal of Food Science \& Technology, vol. 51, no. 3, pp. 710-719, 2016.

[31] S. Hoche, M. A. Hussein, and T. Becker, "Critical process parameter of alcoholic yeast fermentation: speed of sound and density in the temperature range 5-30 ${ }^{\circ} \mathrm{C}$," International Journal of Food Science \& Technology, vol. 49, no. 11, pp. 2441-2448, 2014. 